\title{
A comparison of the effectiveness of amitriptilin and pregabalin treatment in fibromyalgia patients
}

\author{
Gunseli Acet, ${ }^{1}$ Arzu Kaya, ${ }^{2}$ Semra Akturk, ${ }^{3}$ Gurkan Akgol ${ }^{2}$ \\ ${ }^{1}$ Department of Physical Medicine and Rehabilitation, Malatya Training and Research Hospital, Malatya, Turkey \\ 2Department of Physical Medicine and Rehabilitation, Firat University Faculty of Medicine, Elazig, Turkey \\ ${ }^{3}$ Department of Physical Medicine and Rehabilitation, Inonu Univesity Faculty of Medicine, Malatya, Turkey
}

\section{ABSTRACT}

OBJECTIVE: The present study is a comparison of the effectiveness of amitriptyline and pregabalin on the symptoms of fibromyalgia patients.

METHODS: A total of 71 female patients aged $\geq 18$ years were included in this study. The patients were divided into 2 groups. Pregabalin $(n=36)$ or amitriptyline $(n=35)$ treatment was initiated at daily oral dose of $450 \mathrm{mg}$ and $25 \mathrm{mg}$, respectively for the indicated number of patients. The patients were evaluated at the start of treatment and at the end of 12 weeks. The Fibromyalgia Impact Questionnaire, Fatigue Severity Scale, Modified Fatigue Impact Scale, Hospital Anxiety Depression Scale, Nottingham Health Profile, Mini Mental State Test, and the Leeds Assessment of Neuropathic Symptoms and Signs (LANSS) were administered to all study participants. Pain at all tender points was measured using a pressure algometer.

RESULTS: Significant improvement was observed in both groups after 12 weeks of treatment $(p<0.05)$. Percent change in LANSS was greater in the pregabalin group compared with the amitriptyline group. Tender point pressure pain thresholds and total myalgic score improved significantly in both groups $(p<0.05)$; however higher percentage change in these parameters was achieved in the amitriptyline group when compared with the pregabalin group $(\mathrm{p}<0.05)$.

CONCLUSION: Both drugs improved pain, fatigue, sleep disorder, disability, psychological evaluation, and cognitive function; however, amitriptyline was more effective at reducing experimentally measured pain than neuropathic pain. According to these results, preference for pregabalin may be recommended in fibromyalgia patients whose primary complaint is neuropathic pain.

Keywords: Amitriptyline; fibromyalgia; neuropathic pain; pregabalin; pressure pain threshold.

Received: March 20, 2017 Accepted: July 04, 2017 Online: August 26, 2017

Correspondence: Dr. Semra AKTURK. Turgut Ozal Tip Merkezi, Fiziksel Tedavi ve Rehabilitasyon Klinigi

1. Kat, Malatya, Turkey.

Tel: +90 422 - 3410660 e-mail: semrakayakturk@hotmail.com

(c) Copyright 2017 by Istanbul Northern Anatolian Association of Public Hospitals - Available online at www.kuzeyklinikleri.com 
$\mathrm{F}$ ibromyalgia syndrome (FMS) is a chronic, extra-articular, rheumatic syndrome characterized by widespread musculoskeletal system pain and tender points at specific anatomical points [1]. Its prevalence ranges between $2 \%$ and $7 \%$ in the population [2]. Although trauma, infection, autoimmune, genetic, endocrinological, and emotional factors have been emphasized in the etiology of FMS, its exact etiology is still not known $[3,4]$. Abnormalities in central mechanisms may explain its etiopathogenesis. Sleep disorders, psychological function, disorders in pain modulation, biochemical changes in the central nervous system, and neuroendocrinological dysfunction play roles in abnormalities of central mechanisms [5-8]. Abnormalities in the mechanisms of tryptophan, which is a serotonin precursor, are seen in these patients. As a neuropeptide, substance $\mathrm{P}$ is a primary nociceptive afferent modulator, and plays a role in the regulation of immune functions. In FMS patients, plasma substance $\mathrm{P}$ level is within normal limits; however, the level in cerebrospinal fluid was found to be 3 times higher than that of normal control subjects [9].

A pharmacological agent that completely resolves all symptoms and ensures complete well-being is not yet available for patients with fibromyalgia. Treatments are most frequently targeted at symptoms using non-standardized methods [10]. Depression medications, such as tricyclic antidepressants, selective serotonin reuptake inhibitors, serotonin, and noradrenalin reuptake inhibitors have been demonstrated to be effective at reducing pain, depression, and fatigue in patients with fibromyalgia [11]. Tricyclic antidepressants are believed to be effective in fibromyalgia treatment by influencing the reuptake of amines, such as serotonin and epinephrine, by inducing the production of endogenous opioids, and regulating central motor activity, thereby improving symptoms of depression. Amitriptyline is an important member of this class of antidepressants. Its analgesic mechanism has not been fully explained yet; however, it has been demonstrated to have greater effectiveness in providing relief for patients with fibromyalgia than selective serotonin reuptake inhibitors, independent of its antidepressant effects. It has been proposed that the inhibition of noradrenaline reuptake, N-Methyl-D-aspartic acid receptor antagonism, and the blockade of muscarinic receptors and ion channels may contribute to the analgesic effect of amitriptyline [12-14].

It has been demonstrated in various studies that pregabalin is effective in the treatment of fibromyalgia [15-17]. In 2007, it was the first medicine approved by the US Food and Drug Administration for fibromyalgia treatment. It exerts its effect by binding to alpha-2 delta subunits of voltage-sensitive calcium channels and slowing the entry of calcium into neurons It does not interact with benzodiazepine or gamma-aminobutyric acid (GABA)-A and GABA-B receptors. Since it does not bind to these receptor sites, it also does not interact with other antiepileptics, antidepressants, or analgesics, and it can be used safely in drug combinations [16].

The aim of this study was to compare the effectiveness of amitriptyline, with established efficacy in patients with fibromyalgia, with that of pregabalin, the use of which in the treatment of fibromyalgia has become increasingly prevalent in recent years.

\section{MATERIALS AND METHODS}

The present study was approved by the Firat University Faculty of Medicine ethics committee and was conducted in accordance with the Declaration of Helsinki. Written, informed consent was obtained from all participants.

Among admissions to Firat University Hospital Physical Medicine and Rehabilitation outpatient clinic, a total of 71 female patients aged $\geq 18$ years (mean age: $39.2 \pm 8.96$ years in the amitriptyline group and $36.5 \pm 6.68$ years in the pregabalin group) having predominantly neuropathic pain pattern diagnosed as FMS based on the diagnostic criteria of the American College of Rheumatology were included in this study. Patients who had previously been operated on for lumbar herniated disc, those who had recently received physical therapy, or pregabalin or antidepressant treatment within the previous 2 months, patients with inflammatory or malignant diseases, pregnant patients, cases with severe psychological disorders or systemic disease were not enrolled in the study. 
Demographic data (age, gender, marital status, socioeconomic status, type of employment, educational level, smoking/alcohol use, body mass index) of the patients were recorded. All participants underwent systemic examination and laboratory assessment of complete blood count, routine biochemical analysis, erythrocyte sedimentation rate, rheumatoid factor, C-reactive protein level, presence of Brucella, and thyroid function was performed. Body mass index was measured using Tanita BC418MA scale with Segmental Body Composition Analyzer (Tanita Corp., Tokyo, Japan).

The patients were randomly divided into 2 groups. Pregabalin $(n=36)$ or amitriptyline $(n=25)$ therapy was initiated at daily $150 \mathrm{mg}$ and slowly increased to $450 \mathrm{mg}$ oral dose, and $10 \mathrm{mg}$ to $25 \mathrm{mg}$, respectively, in the indicated number of patients. The patients were evaluated monthly over the course of 3-month treatment for effects using the Visual Analogue Scale (VAS) for pain, assessment of sleep quality, and side effects of the treatment.

At baseline and at the conclusion of 12 weeks, clinical evaluations were performed in both groups using several measurement tools. Functional evaluation was performed with the Fibromyalgia Impact Questionnaire (FIQ), impact of fatigue on daily activities was measured with the Fatigue Severity Scale (FSS) and the Modified Fatigue Impact Scale (MFIS), quality of life was assessed with the Nottingham Health Profile (NHP), risk for anxiety and depression was evaluated with the Hospital Anxiety Depression Scale (HADS), cognitive function was measured with the Mini Mental State Examination (MMSE), neuropathic pain was gauged using the Leeds Assessment of Neuropathic Symptoms and Signs (LANSS), and a global scoring of severity of pain estimated separately by the patient and the physician using the VAS. VAS and sleep quality evaluations were performed at 4-week intervals.

Pressure pain threshold (PPT) measurements were taken from tender points (18 points), and control points ( 3 points: midpoint of the forehead; distal one-third dorsal aspect of the dominant forearm, and nail of the thumb of the dominant hand) before and after the treatment using a manual algometry device (Force Dial model FDK 40 Push
Pull Force Gage; Wagner Instruments, Riverside, CT, USA). Pressure was applied with $1 \mathrm{~kg}$ increase per second. Skin temperature was measured using Scantemp Pro 440 infrared thermometer (Cardinal Health, Inc, Dublin, OH, USA).

The FIQ was administered to measure the health status of the patients. This disease-specific measure of global health status has been validated for a Turkish population with FMS [18]. The FIQ is a 10 -item, self-administered test that measures physical function, work status, depression, anxiety, sleep, pain, stiffness, fatigue, and well-being. As score approaches maximum total of 100 it indicates more severe symptoms and disability.

The FSS is the most frequently used questionnaire to evaluate the effect of fatigue on daily activities. Validation and reliability studies for Turkish version were performed by Armutlu et al. The scale consists of 9 statements, each of which is scored between 1 (I don't agree at all), and 7 (I strongly agree). The FSS score is the average value of score for all 9 statements. A higher score reflects a more severe state of fatigue $[19,20]$.

The HADS is a self-assessment scale used to determine risks for, level of, and changes in severity of anxiety and depression. It comprises a total of 14 questions, 7 related to anxiety and 7 concerning depression.

Health-related quality of life of the patients was evaluated with the NHP, which contains 38 items in 6 domains related to level of energy (3 items), pain (8 items), emotional reactions ( 9 items), sleep ( 5 items), physical mobility ( 8 items), and social isolation ( 5 items). Items in each domain are assigned a weight; the total score for each domain is 100; a score of 0 indicates good subjective health status, while 100 indicates poor subjective health status. Total NHP total score is obtained by averaging the 6 domain scores.

The LANSS scale evaluates symptoms of neuropathic pain and responsiveness to treatment. Sensitivity and specificity in the discrimination between neuropathic and non-neuropathic pain have been evaluated in a local patient population [21].

Functions of orientation, registration, attention 
TABLE 1. Comparison of parameters in the amitriptyline group

\begin{tabular}{lccc} 
Parameters & Before treatment & After treatment (3 months) & $\mathrm{p}$ \\
\hline VAS-pain & $7.77 \pm 1.65$ & $3.37 \pm 1.76$ & $\mathbf{0 . 0 0}$ \\
FIQ-pain & $7.72 \pm 1.71$ & $3.56 \pm 1.82$ & $\mathbf{0 . 0 0}$ \\
FIQ-morning fatigue & $8.32 \pm 1.34$ & $3.39 \pm 2.31$ & $\mathbf{0 . 0 0}$ \\
FSS & $5.77 \pm 1.04$ & $4.14 \pm 1.28$ & $\mathbf{0 . 0 0}$ \\
NHP-fatigue & $91.86 \pm 23.68$ & $33.55 \pm 37.92$ & $\mathbf{0 . 0 0}$ \\
NHP pain & $66.22 \pm 26.29$ & $24.64 \pm 15.46$ & $\mathbf{0 . 0 0}$ \\
FIQ-anxiety & $6.15 \pm 2.54$ & $1.25 \pm 1.79$ & $\mathbf{0 . 0 0}$ \\
FIQ-depression & $5.81 \pm 2.68$ & $1.29 \pm 1.66$ & $\mathbf{0 . 0 0}$ \\
NHP-physical activity & $34.51 \pm 28.18$ & $8.55 \pm 14.18$ & $\mathbf{0 . 0 0}$ \\
NHP-sleep & $54.46 \pm 33.70$ & $11.84 \pm 16.45$ & $\mathbf{0 . 0 0}$ \\
NHP-social isolation & $26.41 \pm 37.13$ & $7.1413 \pm 13.28$ & $\mathbf{0 . 0 0 1}$ \\
NHP-emotional reaction & $10.97 \pm 19.75$ & $2.15 \pm 3.88$ & $\mathbf{0 . 0 0 5}$ \\
HADS-depression & $8.94 \pm 2.90$ & $6.94 \pm 1.96$ & $\mathbf{0 . 0 0 0}$ \\
HADS-anxiety & $10.17 \pm 2.06$ & $8.20 \pm 1.90$ & $\mathbf{0 . 0 0 0}$ \\
\hline
\end{tabular}

FIQ: Fibromyalgia Impact Questionnaire; FSS: Fatigue Severity Scale; HADS: Hospital Anxiety and Depression Scale; NHP: Nottingham Health Profile; VAS: Visual Analogue Scale.

and calculation, recall, and language are measured with the MMSE questionnaire, which consists of 11 questions and has a total possible score of 30 points. Score $<24$ points indicates cognitive impairment [22-24].

\section{Statistical analysis}

Statistical analyses were performed using SPSS for Windows, Version 15.0 (SPSS Inc., Chicago, IL, USA). Descriptive statistics were used to summarize the distribution of patient and control group demographic characteristics. For intragroup comparisons of measurements performed at baseline and after 12 weeks of therapy, parametric Student's t-test was used. McNemar's test was used for intragroup comparisons of rates, and intergroup comparisons were performed using parametric Student's ttest. For intergroup comparisons of measurements with ordinal and nominal variables, chi-square test was used. Pairwise comparisons of multiple measurements were assessed with Student's t-test, with a limit of significance of $0.016(0.05 /$ number of comparisons $[n=3]=0.016)$. For other comparisons, limit of significance was accepted as $\mathrm{p}=0.05$.

\section{RESULTS}

A total of 66 patients (amitriptyline group, $n=32$; pregabalin group, $n=34$ ) completed the study. No significant intergroup difference was found with respect to age, body mass index, or level of formal education ( $p>0.05)$.

Pre- and post-treatment measurements of VAS and MFIS physical, cognitive, and psychosocial function subscale scores were significantly different in the amitriptyline group (Group 1) $(p=0.00)$. NHP, FIQ, and VAS scores of the patients in the amitriptyline group are provided in Table 1. A significant difference was seen in post-treatment LANSS score, or measurement of anxiety and depression parameters of the patients in Group 1 $(p<0.05)$. No significant difference was found between pre- and post-treatment MMSE scores or sum of 3 PPT control point scores ( $p>0.05)$.

In the pregabalin group, significant differences were observed in the patient self-assessment and the physician's global evaluation (VAS) of the patient, and in the pain subscale scores of the NHP and FIQ $(p<0.05)$. Group scores are provided in 
TABLE2. Comparison of parameters in the pregabalin group

\begin{tabular}{lccc} 
Parameters & Pre-treatment & Post-treatment (3 months) & $\mathrm{p}$ \\
\hline VAS & $8.04 \pm 1.44$ & $4.27 \pm 1.83$ & $\mathbf{0 . 0 0 0}$ \\
NHP-pain & $72.70 \pm 21.14$ & $30.20 \pm 15.85$ & $\mathbf{0 . 0 0 0}$ \\
FIQ-pain & $7.91 \pm 1.65$ & $4.50 \pm 1.80$ & $\mathbf{0 . 0 0 0}$ \\
NHP-pain & $78.37 \pm 35.32$ & $34.44 \pm 38.21$ & $\mathbf{0 . 0 0 0}$ \\
FIQ-fatigue & $8.33 \pm 1.72$ & $4.14 \pm 1.64$ & $\mathbf{0 . 0 0 0}$ \\
FIQ-morning fatigue & $8.04 \pm 2.10$ & $3.67 \pm 1.78$ & $\mathbf{0 . 0 0 0}$ \\
NHP-physical activity & $38.57 \pm 25.59$ & $16.53 \pm 20.51$ & $\mathbf{0 . 0 0 0}$ \\
NHP-sleep & $33.60 \pm 35.64$ & $10.72 \pm 18.73$ & $\mathbf{0 . 0 0 0}$ \\
NHP-social isolation & $12.06 \pm 19.97$ & $5.54 \pm 18.46$ & 0.071 \\
NHP-emotional reactions & $5.60 \pm 11.09$ & $2.80 \pm 9.82$ & 0.174 \\
HADS-depression & $8.36 \pm 2.63$ & $7.02 \pm 2.08$ & $\mathbf{0 . 0 0 0}$ \\
HADS-anxiety & $9.41 \pm 1.93$ & $7.26 \pm 1.60$ & $\mathbf{0 . 0 0 0}$ \\
FIQ-anxiety & $5.94 \pm 2.94$ & $1.67 \pm 1.95$ & $\mathbf{0 . 0 0 0}$ \\
FIQ-depression & $4.91 \pm 3.076$ & $1.60 \pm 1.74$ & $\mathbf{0 . 0 0 0}$ \\
MFIS-physical function & $21.80 \pm 6.54$ & $14.32 \pm 5.04$ & $\mathbf{0 . 0 0 0}$ \\
MFIS-cognitive function & $21.91 \pm 7.85$ & $14.73 \pm 5.66$ & $\mathbf{0 . 0 0 0}$ \\
\hline
\end{tabular}

FIQ: Fibromyalgia Impact Questionnaire; FSS: Fatigue Severity Scale; HADS: Hospital Anxiety and Depression Scale; MFIS: Modified Fatigue Impact Scale; NHP: Nottingham Health Profile; VAS: Visual Analogue Scale.

TABLE 3. Comparison of pretreatment and post-treatment clinical parameters between the amitriptyline and pregabalin groups

Pre-treatment

Post-treatment

\begin{tabular}{lccccccc}
\cline { 2 - 3 } \cline { 6 - 7 } Parameters & Amitiriptyline $(\mathrm{n}=35)$ & Pregabalin $(\mathrm{n}=36)$ & $\mathrm{p}$ & & Amitiriptyline $(\mathrm{n}=32)$ & Pregabalin $(\mathrm{n}=34)$ & $\mathrm{p}$ \\
\hline VAS & $7.42 \pm 1.67$ & $8.04 \pm 1.44$ & $*$ & $4.26 \pm 1.93$ & $4.27 \pm 1.83$ & $*$ \\
NHP-pain & $66.22 \pm 26.29$ & $72.70 \pm 21.14$ & $*$ & $24.64 \pm 15.46$ & $30.20 \pm 15.85$ & $*$ \\
FIQ-pain & $7.72 \pm 1.71$ & $7.91 \pm 1.65$ & $*$ & $3.56 \pm 1.82$ & $4.50 \pm 1.80$ & $\mathbf{0 . 0 4}$ \\
MFIS-pf & $21.22 \pm 6.66$ & $21.80 \pm 6.54$ & $*$ & $14.62 \pm 4.46$ & $14.32 \pm 5.043$ & $*$ \\
NHP-fatigue & $91.86 \pm 23.68$ & $78.37 \pm 35.32$ & $*$ & $33.55 \pm 37.92$ & $34.44 \pm 38.21$ & $*$ \\
FIQ-fatigue & $8.41 \pm 1.62$ & $8.3333 \pm 1.72$ & $*$ & $3.9375 \pm 1.79$ & $4.14 \pm 1.64$ & $*$ \\
NHP-pa & $34.51 \pm 28.18$ & $38.57 \pm 25.59$ & $*$ & $8.55 \pm 14.18$ & $16.53 \pm 20.51$ & $*$ \\
HADS-depression & $8.94 \pm 2.90$ & $8.36 \pm 2.63$ & $*$ & $6.94 \pm 1.96$ & $7.02 \pm 2.08$ & $*$ \\
HADS-anxiety & $10.17 \pm 2.06$ & $9.41 \pm 1.93$ & $*$ & $8.20 \pm 1.90$ & $7.26 \pm 1.60$ & $\mathbf{0 . 0 3 1}$ \\
MMSE & $24.82 \pm 3.15$ & $25.44 \pm 2.96$ & $*$ & $25.56 \pm 3.25$ & $26.32 \pm 2.55$ & $*$ \\
\hline
\end{tabular}

${ }^{*} \mathrm{p}>0.05$. FIQ: Fibromyalgia Impact Questionnaire; FSS: Fatigue Severity Scale; HADS: Hospital Anxiety and Depression Scale; MFIS: Modified Fatigue Impact Scale; MMSE: Mini Mental State Examination; NHP: Nottingham Health Profile; pa: Physical activity; pf: Physical function; VAS: Visual Analogue Scale.

Table 2. Pre- and post-treatment LANSS neuropathic pain scale scores were significantly different in the pregabalin group $(\mathrm{p}=0.00)$. A significant difference was also found in pre- and post-treatment 
TABLE 4. Percent change in tender point and total myalgic scores between groups

\begin{tabular}{lccr} 
Parameters & Amitriptyline $(\mathrm{n}=35)$ & Pregabalin $(\mathrm{n}=36)$ & $\mathrm{P}$ \\
\hline Percent change tender point score & $-0.197 \pm 0.14(-0.61-0.09)$ & $-0.098 \pm 0.12(-0.47-0.17)$ & $\mathbf{0 . 0 0 5}$ \\
Percent change total myalgic score & $-0.160 \pm 0.13(-0.58-0.06)$ & $-0.079 \pm 0.11(-0.44-0.16)$ & $\mathbf{0 . 0 1 2}$
\end{tabular}

total PPT score of tender points and total myalgic score $(\mathrm{p}<0.05)$.

A significant intergroup difference was seen in post-treatment assessments of the FIQ pain subscale, and the HADS anxiety measurement score $(p<0.05)$. Pre- and post-treatment VAS and NHP pain subscale score was not significantly different between groups $(p>0.05)$. Pre- and post-treatment NHP, FIQ, modified fatigue impact scale and VAS scores are presented in Table 3. No significant difference was found between pre- and post-treatment LANSS score $(p>0.05)$, while the percent change was significantly different between groups $(p<0.05)$; a greater degree of improvement was seen in the pregabalin group compared with the amitriptyline group. In the tender point pressure and total myalgic score percent change, however, there was a significant difference between the groups in favor of the amitriptyline group $(p<0.05)$ (Table 4$)$. No significant intergroup difference was found in the rate of pre- and post-treatment symptoms associated with FMS ( $p>0.05)$.

The most frequently reported side effects of all patients in both groups were dizziness and sleep disorders. Dizziness was more frequently seen in the pregabalin group (Figure 1). Changes over time in side effects experienced and VAS scores can be seen in Table 5.

\section{DISCUSSION}

In the present study we observed that the effects of both pregbalin and amitriptyline were similar with respect to effectiveness on pain, fatigue, sleep disorders, disability, psychological factors, and cognitive function; however, amitriptyline was more effective at relieving experimental pain, while pregabalin was

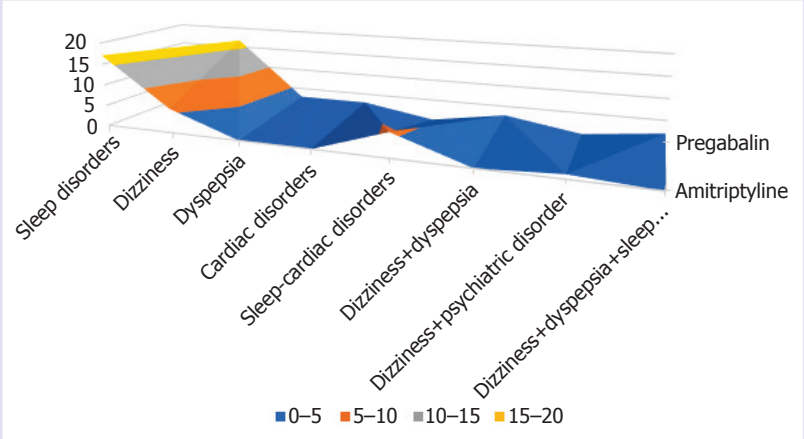

FIGURE 1. Side effects of pregabalin and amitriptyline.

more helpful for neuropathic pain.

Since fibromyalgia patients describe widespread clinical manifestations, an overlap with neuropathic pain has been suggested. Hyperalgesia, allodynia, and paresthesia seen in neuropathic pain can also be observed in FMS. In almost all of these patients, impairment of central pain mechanism, neuroendocrinological abnormalities, and abnormal activation in the nociceptive domain of the brain are seen. These multiple abnormalities strongly suggest that fibromyalgia is a neuropathic pain syndrome $[25,26]$.

A serotonin deficiency proposed in the etiology of FMS may account for the pain and sleep disorders observed, which are important features of this syndrome [27]. Most frequently, antidepressants are used in the treatment of fibromyalgia, and among them the tricyclic antidepressant amitriptyline is most often administered. Generally, the analgesic effects of tricyclic antidepressants are accepted to be an acute effect independent of antidepressant effects, and only require a low dose [28].

Gabapentin, which is an anticonvulsant also used to treat neuropathic pain, was compared with amitriptyline, and both drugs were found to be ef- 
TABLE 5. Assessment of side effects and VAS (pain and sleep) changes in the groups at first, second, and third month

\begin{tabular}{|c|c|c|c|c|c|c|}
\hline \multirow[b]{2}{*}{ Parameters } & \multicolumn{2}{|c|}{ First month } & \multicolumn{2}{|c|}{ Second month } & \multicolumn{2}{|c|}{ Third month } \\
\hline & $\begin{array}{c}\text { Amitiriptyline } \\
\%\end{array}$ & $\begin{array}{c}\text { Pregabalin } \\
\%\end{array}$ & $\begin{array}{c}\text { Amitiriptyline } \\
\%\end{array}$ & $\begin{array}{c}\text { Pregabalin } \\
\%\end{array}$ & $\begin{array}{c}\text { Amitiriptyline } \\
\%\end{array}$ & $\begin{array}{c}\text { Pregabalin } \\
\%\end{array}$ \\
\hline Sleep disorders & 17.1 & 5.6 & 15.2 & 2.7 & 8.3 & 2.7 \\
\hline Dizziness & 5.7 & 16.7 & 5.7 & 13.8 & 2.8 & 6.2 \\
\hline Dyspepsia & 0 & 2.8 & 0 & 2.8 & 0 & 2.8 \\
\hline Cardiac disorders & 0 & 0 & 2.8 & 0 & 2.8 & 0 \\
\hline Sleep+cardiac disorders & 5.7 & 0 & 5.7 & 0 & 5.7 & 0 \\
\hline \multirow[t]{2}{*}{ Dizziness+dyspepsia+sleep disorders } & 0 & 5.6 & 0 & 5.6 & 0 & 5.6 \\
\hline & \multicolumn{2}{|l|}{$\mathrm{p}$} & \multicolumn{2}{|c|}{$\mathrm{p}$} & \multicolumn{2}{|l|}{$\mathrm{p}$} \\
\hline Pregabalin VAS (pain) & 0.00 & 0.00 & 0.00 & 0.00 & 0.00 & 0.00 \\
\hline Pregabalin VAS (sleep) & 0.00 & 0.00 & 0.00 & 0.00 & 0.00 & 0.00 \\
\hline Amitiriptyline VAS (pain) & 0.00 & 0.00 & 0.00 & 0.00 & 0.00 & 0.00 \\
\hline Amitiriptyline VAS (sleep) & 0.00 & 0.00 & 0.00 & 0.00 & 0.00 & 0.00 \\
\hline
\end{tabular}

VAS: Visual Analogue Scale.

fective in pain management. Gabapentin was found to be more effective on paroxysmal stabbing pain [29]. The effect of pregabalin on chronic pain syndromes has been reported to be comparable to that of gabapentin [14]. A study of 529 patients with fibromyalgia investigated the effect of pregabalin on pain, fatigue, and quality of life. Daily dose of $150 \mathrm{mg}, 300 \mathrm{mg}$, and $450 \mathrm{mg}$ pregabalin was compared with a placebo, and at the end of 8 weeks of treatment, a decrease in all painful sensations was reported in all of the pregbalin groups. It was reported that in patients using daily dose of $450 \mathrm{mg}$ pregabalin, painful sensations decreased more dramatically compared with the other groups, and at daily dose of $300 \mathrm{mg}$ and $450 \mathrm{mg}$, sleep quality and fatigue improved markedly relative to the placebo group [16].

In our study, in both the pregabalin and amitriptyline groups, considerable improvement was seen in parameters of pain; however, the percent of change, which indicated decrease in the severity of pain, did not differ significantly between groups. The present study included patients with prominent neuropathic complaints. The LANSS was used to evaluate neuropathic pain pattern. LANSS scores improved significantly in both the amitriptyline and pregabalin group, yet intergroup comparisons revealed greater improvement in the pregabalin group.

Morning fatigue is found in $75 \%$ to $80 \%$ of patients with fibromyalgia [30-32], though severity varies. A similar percentage of patients complains of poor sleep or sleep disorders [33]. Antidepressants demonstrate important effects on sleep quality and the fatigue seen in FMS [1]. An important difference in the NHP sleep subscale scores was observed with respect to restless sleep in both amitriptyline and pregabalin users. Neither treatment was superior in terms of improvement in sleep problems or morning fatigue.

In another study performed to investigate the effect of pregabalin on anxiety disorders, effectiveness in the short term was demonstrated relative to placebo. Pregabalin was also found to be more effective in patients who partially responded to atypical antipsychotic drug quetiapine [34]. In our study, we observed comparable, favorable effects for both pregabalin and amitriptyline on anxiety and depression in patients with fibromyalgia. 
The most important side effects of pregabalin are somnolence and dizziness. They are typically observed within the first 1 to 2 weeks of starting the drug therapy, and are more marked among the elderly $[17,35]$. Among the most frequently seen side effects of tricyclic antidepressants are anticholinergic effects, such as dry mouth and constipation, and postural hypotension, sedation, and weight gain [36]. In our study, consistent with literature findings, the most frequently seen side effects of pregabalin users were dizziness (8.5\%) and sleep disorders $(2.8 \%)$; however, none of the patients discontinued the treatment due to these side effects. In the amitriptyline group, the most frequent side effects were sleep disorders (8.5\%) and dizziness (2.8\%). Dizziness was more common in the pregabalin group than the amitriptyline group. Generally, tolerance developed in 2 to 3 weeks, and these complaints decreased at control visits.

In our study, PPTs and skin temperature were measured at all fibromyalgia control and tender points using an algometer and an infrared thermometer, respectively. In both the amitriptyline and pregabalin groups, control point scores did not change after treatment relative to baseline, while tender point and total myalgia scores improved in both groups. Tender point PPTs, which are considered a laboratory (experimental) assessment of pain, decreased more dramatically in the amitriptyline group after the treatment.

The main limitation of this study is the small number of participants and the inability to prolong the follow-up period due to strict exclusion criteria of the study.

In conclusion, based on the data we gathered in this study, we can say that neither drug is superior in terms of effectiveness on systemic symptoms of myalgia. However, we can state that amitriptyline is more effective in decreasing experimental pain, whereas pregabalin is more effective in relieving neuropathic complaints. Accordingly, a preference for pregabalin may be recommended for FMS patients primarily complaining of neuropathic pain.

Conflict of Interest: None declared.
Financial Disclosure: The authors declared that this study has received no financial support.

Authorship contributions: Concept - G.Acet, A.K. Data collection \&/or processing - G.A; Analysis and/or interpretation - G.Acet; Writing - S.A.

\section{REFERENCES}

1. Koçanoğulları H. Fibromiyalji sendromu. Gümüşdiş G, Doğanavşargil E, editors. Klinik Romatoloji. İstanbul: Deniz Press; 1999. p. 549-54.

2. Branco JC, Bannwarth B, Failde I, Abello Carbonell J, Blotman F, Spaeth M, et al. Prevalence of fibromyalgia: a survey in five European countries. Semin Arthritis Rheum 2010;39:448-53.

3. Yunus MB. Towards a model of pathophysiology of fibromyalgia: aberrant central pain mechanisms with peripheral modulation. J Rheumatol 1992;19:846-50.

4. Reisine S, Fifield J, Walsh SJ, Feinn R. Do employment and family work affect the health status of women with fibromyalgia? J Rheumatol 2003;30:2045-53.

5. Moldofsky H, Scarisbrick P, England R, Smythe H. Musculosketal symptoms and non-REM sleep disturbance in patients with "fibrositis syndrome" and healthy subjects. Psychosom Med 1975;37:341-51.

6. Gür A. Etiopathogenesis in Fibromyalgia. Turk J Phys Med Rehab 2008:54 Suppl 1;4-11.

7. Reynolds MD. Clinical diagnosis of psychogenic rheumatism. West J Med 1978;128:285-90.

8. White KP, Nielson WR, Harth M, Ostbye T, Speechley M. Chronic widespread musculoskeletal pain with or without fibromyalgia: psychological distress in a representative community adult sample. J Rheumatol 2002;29:588-94.

9. Russell IJ, Orr MD, Littman B, Vipraio GA, Alboukrek D, Michalek JE, et al. Elevated cerebrospinal fluid levels of substance $\mathrm{P}$ in patients with the fibromyalgia syndrome. Arthritis Rheum 1994;37:1593-601.

10. Özgöçmen S, Ardıçoğlu Ö. Pharmacotherapy in fibromyalgia syndrome. Syndrome 2000;12:84-8.

11. Lee YH, Song GG. Comparative efficacy and tolerability of duloxetine, pregabalin, and milnacipran for the treatment of fibromyalgia: a Bayesian network meta-analysis of randomized controlled trials. Rheumatol Int 2016;36:663-72.

12. Bennet RM. The Fibromyalgia Syndrome. In: Kelley WN. Harris ED Jr. Ruddy S. Sledge C, editors. Textbook of Rheumatology. Philadelphia: WB Saunders Company; 1997. p. 471-9.

13. Patkar AA, Masand PS, Krulewicz S, Mannelli P, Peindl K, Beebe KL, et al. A randomized, controlled, trial of controlled release paroxetine in fibromyalgia. Am J Med 2007;120:448-54.

14. Frank B, Niesler B, Bondy B, Späth M, Pongratz DE, Ackenheil $\mathrm{M}$, et al. Mutational analysis of serotonin receptor genes: HTR3A and HTR3B in fibromyalgia patients. Clin Rheumatol 2004;23:338-44. 
15. White KP, Harth M. Classification, epidemiology, and natural history of fibromyalgia. Curr Pain Headache Rep 2001;5:3209.

16. Crofford LJ, Rowbotham MC, Mease PJ, Russell IJ, Dworkin RH, Corbin AE, et al; Pregabalin 1008-105 Study Group. Pregabalin for the treatment of fibromyalgia syndrome: results of a randomized, double-blind, placebo-controlled trial. Arthritis Rheum 2005;52:1264-73.

17. Calandre EP, Morillas-Arques P, Rodriguez-Lopez CM, RicoVillademoros F, Hidalgo J. Pregabalin augmentation of quetiapine therapy in the treatment of fibromyalgia: an open-label, prospective trial. Pharmacopsychiatry 2007;40:68-71.

18. Sarmer S, Ergin S, Yavuzer G. The validity and reliability of the Turkish version of the Fibromyalgia Impact Questionnaire. Rheumatol Int 2000;20:9-12.

19. Mancuso CA, Rincon M, Sayles W, Paget SA. Psychosocial variables and fatigue: a longitudinal study comparing individuals with rheumatoid arthritis and healthy controls. J Rheumatol 2006;33:1496-502.

20. Anton HA, Miller WC, Townson AF. Measuring fatigue in persons with spinal cord injury. Arch Phys Med Rehabil 2008;89:538-42.

21. Yucel A, Senocak M, Kocasoy Orhan E, Cimen A, Ertas M. Results of the Leeds assessment of neuropathic symptoms and signs pain scale in Turkey: a validation study. J Pain 2004;5:427-32.

22. Çapac1 K, Hepgüler S. Fibromyalgia Syndrome: Diagnosis and Treatment. Ege J of Physical Med and Reh 1999;5:73-82.

23. Crofford LJ. The relationship of fibromyalgia to neuropathic pain syndromes. J Rheumatol Suppl 2005;75:41-5.

24. Arnold LM, Goldenberg DL, Stanford SB, Lalonde JK, Sandhu HS, Keck PE Jr, et al. Gabapentin in the treatment of fibromyalgia: a randomized, double-blind, placebo-controlled, multicenter trial. Arthritis Rheum 2007;56:1336-44.

25. Staud R, Domingo M. Evidence for abnormal pain processing in fibromyalgia syndrome. Pain Med 2001;2:208-15.

26. Martínez-Lavin M, López S, Medina M, Nava A. Use of the leeds assessment of neuropathic symptoms and signs questionnaire in patients with fibromyalgia. Semin Arthritis Rheum 2003;32:407-11.

27. Clifford DB. Treatment of pain with antidepressants. Am Fam Physician 1985;31:181-5.

28. Goldenberg DL, Felson DT, Dinerman H. A randomized, controlled trial of amitriptyline and naproxen in the treatment of patients with fibromyalgia. Arthritis Rheum 1986;29:1371-7.

29. Keskinbora K, Pekel AF, Aydinli I. Comparison of efficacy of gabapentin and amitriptyline in the management of peripheral neuropathic pain. Agri 2006;18:34-40.

30. Kocabas H, Yllmaz G, Uğurlu H. Comparison of Effectiveness of Hydrotherapy and Amitriptyline in FibromyalgiaTreatment. Rheumatism 2007;22:91-6.

31. Çapacı K, Hepgüler S. Comparison of amitriptyline and paroxetine in the treatment of fibromyalgia syndrome. Ege J of Physical Med and Reh 1998;4:27-32.

32. Arnold LM, Russell IJ, Diri EW, Duan WR, Young JP Jr, Sharma U, et al. A 14-week, randomized, double-blinded, placebocontrolled monotherapy trial of pregabalin in patients with fibromyalgia. J Pain 2008;9:792-805.

33. Yunus MB, Masi AT. Fibromyalgia, Restless Legs Syndrome: Periodic Limb Movement Disorder and Psychogenic Pain. In: McCarty DJ, Kopman WJ, editors. Arthritis and Allied Conditions. $12^{\text {th }}$ ed. Philadelphia: Lea\&Febiger; 1992, p. 1383.

34. Feltner DE, Crockatt JG, Dubovsky SJ, Cohn CK, Shrivastava RK, Targum SD, et al. A randomized, double-blind, placebocontrolled, fixed-dose, multicenter study of pregabalin in patients with generalized anxiety disorder. J Clin Psychopharmacol 2003;23:240-9.

35. Häuser W, Walitt B, Fitzcharles MA, Sommer C. Review of pharmacological therapies in fibromyalgia syndrome. Arthritis Res Ther 2014;16:201.

36. Beniczky S, Tajti J, Tímea Varga E, Vécsei L. Evidence-based pharmacological treatment of neuropathic pain syndromes. J Neural Transm (Vienna) 2005;112:735-49. 